\title{
Application des techniques physiques, géochimiques et isotopiques pour l'évaluation de l'infiltration sous climat tempéré
}

\section{Physical, Chemical and isotopic techniques to evaluate recharge under temperate climates}

\author{
A. Adorni-Braccesi, M. Acutis, S. Merlo et G. M. Zuppi
}

Volume 14, numéro 3, 2001

URI : https://id.erudit.org/iderudit/705419ar

DOI : https://doi.org/10.7202/705419ar

\section{Aller au sommaire du numéro}

\section{Éditeur(s)}

Université du Québec - INRS-Eau, Terre et Environnement (INRS-ETE)

ISSN

0992-7158 (imprimé)

1718-8598 (numérique)

\section{Découvrir la revue}

Citer cet article

Adorni-Braccesi, A., Acutis, M., Merlo, S. \& Zuppi, G. M. (2001). Application des techniques physiques, géochimiques et isotopiques pour l'évaluation de l'infiltration sous climat tempéré. Revue des sciences de l'eau / Journal of Water Science, 14(3), 243-263. https://doi.org/10.7202/705419ar

\section{Résumé de l'article}

Depuis plusieurs années, la Zone Non Saturée d'une parcelle expérimentale au nord de l'Italie (plaine du Pô) a fait l'objet de nombreuses études sur les caractéristiques physiques et géologiques du milieu, ainsi que sur les modalités du mouvement des eaux d'infiltration. Le site a été équipé de 6 tensiomètres à mercure, 6 bougies poreuses pour l'échantillonnage de l'eau et d'un tube d'accès pour la sonde à neutrons.

L'application des différentes méthodes d'évaluation a fourni, pour la période d'étude (Septembre 1994-Août 1995), des valeurs de l'infiltration sensiblement différentes. La méthode physique, basée sur le calcul du bilan hydrique annuel, et considérant le sol comme un unique réservoir monocouche, a indiqué que $19 \%$ du total des précipitations arrive à s'infiltrer. D'autre part, un modèle de simulation intégrant les caractéristiques hydrodynamiques du sol a permis d'évaluer la recharge à $29 \%$ des précipitations annuelles. Le calcul de la répartition de la teneur en eau sur le profil porte sur la description des fluctuations temporelles du plan de flux nul, en relation avec les épisodes pluvieux et l'intensité de l'évaporation.

La méthode géochimique du "profil en ions conservés" entre l'eau de pluie et du sol a permis, quant à elle, d'évaluer la lame d'eau infiltrée à environ $12 \%$ des précipitations annuelles.

La composition isotopique des eaux extraites du sol manifeste un enrichissement global en isotopes lourds $\left({ }^{2} \mathrm{H}\right.$ et $\left.{ }^{18} \mathrm{O}\right)$ par rapport aux eaux météoriques, conséquence d'une évaporation intense. L'infiltration semble être de type "piston flow" dispersif. Elle suit, seulement pendant l'hiver, un flux où la composante convective dans la microporosité est prédominante sur la composante diffusive qui a lieu, de préférence, dans la macroporosité. 


\title{
Application des techniques physiques, géochimiques et isotopiques pour l'évaluation de l'infiltration sous climat tempéré
}

\author{
Physical, chemical and isotopic techniques \\ to evaluate recharge under temperate climates
}

\section{A. ADORNI-BRACCESI ${ }^{1}$, M. ACUTIS $^{2}$, S. MERLO ${ }^{1}$, G.M. ZUPPI ${ }^{1,3,}$ *}

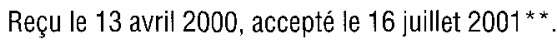

\section{SUMMARY}

In groundwater management, it is necessary to define water movement, evaluate infiltration and evapotranspiration rates, and quantify the physico-chemical evolution of transported solutes throughout the unsaturated zone (UZ). Traditionally, in temperate regions, recharge rate is evaluated by the comparison between physical methods (based on direct measurements of hydrological parameters with lysimeters, tensiometers and neutron gauges) and geochemical approaches (conservative ions, stable isotope profiles and artificial tracers).

In the Po Plain (Northern Italy) intensive agricultural irrigation and overexploitation of groundwater deeply affect the quality and availability of shallow groundwater resources. The region has been intensively cultivated with corn and rice during the last 5 centuries. Irrigation canals have been constructed in order to distribute water from alpine rivers to areas more favourable to agriculture. In the past, only the water balance method has been applied to obtain recharge rates in these situations.

An experimental field site has been operational since 1987 in the ENEAEUREX Nuclear Centre of Saluggia. The experimental plot represents an unsaturated zone in fluvio-glacial deposits (Holocene) of the Dora Baltea River, formed by gravel and sand, interlayered with silt levels. The water table is usually at a depth of $200 \mathrm{~cm}$ but it varies as a function of the river level. The objectives of the present study were to describe water movement throughout the $U Z$ and to evaluate infiltration and evapotranspiration rates

. Dipartimento di Scienze della Terra, Università di Torino, Via Valperga Caluso 35, 10125 Torino, Italia. Present address : R.M.C. S.r.I., Corso Italia 69, 56125 Pisa, Italia.

2. Dipartimento di Ingegneria Agraria ed Agronomia del Territorio, Università Federico II, Via Università 100, 80055 Portici (NA), Italia.

3. Dipartimento di Scienze Ambientali, Università Ca' Foscari, Calle Larga Santa Marta, Dorsoduro 2137, 30123 Venezia, Italia.

* Correspondance. E-mail : zuppi@tin.it

** Les commentaires seront reçus jusqu'au 14 août 2002. 
using different physico-chemical methodologies. The Saluggia plot was equipped with six tensiometers, a neutron gauge hole and 6 porous cups, up to a depth of $160 \mathrm{~cm}$.

During 10 years of monitoring, the most favourable period for understanding infiltration processes and water movement through the $\mathrm{UZ}$ is September 1994-August 1995. This period is characterised by an exceptional rain event (300 mm in 48 hours), followed by a river flood. Water samples collected with porous cups at various depths have been analysed for chemistry and stable isotope composition, together with rainfall and Dora Baltea River water.

In October 1994 (beginning of the rainy period) and May 1995 (beginning of the dry period) soil samples were collected at different depths and water was extracted under vacuum conditions. Stable isotope analyses were performed on extracted waters. Soil samples were also analysed for granulometry, chemical and mineralogical composition.

Reducing soil to an unique monolayer aquifer, the water balance method suggests that only $19 \%$ of the total precipitation infiltrates, whereas a simulation model taking into account soil hydrodynamic characteristics estimates an infiltration rate of $29 \%$ of the rainfall. The position of the Zero Flux Plane (ZFP) fluctuates seasonally between the surface and the maximum studied depth, as a consequence of precipitation events and evaporation fluxes. In some periods, the ZFP is level with the water table and direct discharge may occur.

Measurements of the stable isotopic composition $\left(\delta^{2} \mathrm{H}\right.$ and $\left.\delta^{18} \mathrm{O}\right)$ of soil water allows a quantitative estimate of direct groundwater discharge. On a $\delta^{2} H$ versus $\delta^{18} \mathrm{O}$ plot, pore waters from the $\mathrm{UZ}$ have an isotopic composition that differs from that of the majority of groundwater samples, plotting below the local rain water line and indicating some degree of evaporation during the recharge process. The isotopic enrichment is particularly significant at the evaporation front, suggesting that pore water in soil reflects a different recharge regime from those of the regional ground waters. Water isotope and solute composition were substantially modified from their original composition during the infiltration process within the soil, via mechanisms such as anion exchange with soil particles, salt precipitation/dissolution or isotopic fractionation.

Areal recharge was also evaluated using the depth distribution of a conservative solute. Assuming that chloride is derived from precipitation alone, $\mathrm{Cl}^{-}$ content in soil and in rain water suggests that $12 \%$ of total rainfall infiltrates. Therefore, the recharge rate is estimated to be less than $100 \mathrm{~mm} / \mathrm{a}$. These data are in agreement with other results obtained by the chloride concentration profile method, in areas of the Po Valley, but are considerably different from those evaluated by the traditional physical methods.

Key-words: unsaturated zone, temperate climate, recharge, water balance, conservative ions, isotopic composition, microporosity, macroporosity.

\section{RÉSUMÉ}

Depuis plusieurs années, la Zone Non Saturée d'une parcelle expérimentale au nord de l'Italie (plaine du Pô) a fait l'objet de nombreuses études sur les caractéristiques physiques et géologiques du milieu, ainsi que sur les modalités du mouvement des eaux d'infiltration. Le site a été équipé de 6 tensiomètres à mercure, 6 bougies poreuses pour l'échantillonnage de l'eau et d'un tube d'accès pour la sonde à neutrons.

L'application des différentes méthodes d'évaluation a fourni, pour la période d'étude (septembre 1994-août 1995), des valeurs de l'infiltration sensible- 
ment différentes. La méthode physique, basée sur le calcul du bilan hydrique annuel, et considérant le sol comme un unique réservoir monocouche, a indiqué que $19 \%$ du total des précipitations arrive à s'infiltrer. D'autre part, un modèle de simulation intégrant les caractéristiques hydrodynamiques du sol a permis d'évaluer la recharge à $29 \%$ des précipitations annuelles. Le calcul de la répartition de la teneur en eau sur le profil porte sur la description des fluctuations temporelles du plan de flux nul, en relation avec les épisodes pluvieux et l'intensité de l'évaporation.

La méthode géochimique du «profil en ions conservés » entre l'eau de pluie et du sol a permis, quant à elle, d'évaluer la lame d'eau infiltrée à environ $12 \%$ des précipitations annuelles.

La composition isotopique des eaux extraites du sol manifeste un enrichissement global en isotopes lourds $\left({ }^{2} \mathrm{H}\right.$ et $\left.{ }^{18} \mathrm{O}\right)$ par rapport aux eaux météoriques, conséquence d'une évaporation intense. L'infiltration semble être de type « piston flow » dispersif. Elle suit, seulement pendant l'hiver, un flux où la composante convective dans la microporosité est prédominante sur la composante diffusive qui a lieu, de préférence, dans la macroporosité.

Mots clés : zone non saturée, climat tempéré, infiltration, bilan hydrique, ions conservatifs, composition isotopique, microporosité, macroporosité.

\section{1 - INTRODUCTION}

La gestion rationnelle et le contrôle de la qualité des ressources en eau dans les régions à agriculture intensive demandent de décrire les modalités du mouvement de l'eau dans la Zone non saturée (ZNS), de quantifier le bilan entre l'infiltration et la reprise évapotranspiratoire et de définir les transformations physicochimiques ayant lieu dans le sol.

Les méthodes à disposition des hydrologues pour évaluer la quantité d'eau véhiculée à travers le sol sont celles fondées sur les formules empiriques tirées des paramètres climatiques et hydrologiques et appliquées surtout à l'échelle régionale ou kilométrique (THORNTHWAITE et MATHER, 1957). À celles-ci s'ajoutent les méthodes relativement récentes valables à l'échelle locale, basées sur l'interprétation des profils physiques, des profils géochimiques et sur la composition isotopique de l'eau dans le sol (ALLISON, 1988 ; DARLING et BATH, 1988 ; FONTES et EDMUNDS, 1989 ; SAXENA et DRESSIE, 1984). L'évaluation de l'infiltration ou des pertes par évapotranspiration avec ces méthodes est plus facile en région sous climat extrême, surtout en zone aride et semi-aride (ROZANSKI et al., 1997), qu'en région sous climat tempéré. En effet, dans les premières, les signaux météoriques sont très rares et bien différenciés dans le temps ; l'infiltration est alors mieux marquée et peut être suivie en détail. En revanche, la fréquence des précipitations en zone tempérée et humide se traduit par l'homogénéisation des signaux à profondeurs variables selon la conductivité hydraulique du milieu.

Depuis longtemps, en régions sous climat tempéré, l'évaluation de la recharge est basée sur une comparaison entre les différentes méthodes précédemment citées (ALLISON, 1988). Dans le cas de la plaine du Pô, jusqu'à présent, seul le bilan hydrique était utilisé pour évaluer la réalimentation naturelle 
des nappes et les relations entre les eaux de surface et les eaux souterraines (CNR-IRSA, 1979 ; 1981).

Une parcelle expérimentale dans le nord de l'Italie, pour laquelle on dispose d'enregistrements des données physiques et chimiques sur une longue durée, est située près du centre de recherche ENEA-EUREX de Saluggia. Au cours de dix années (1986-1995) d'observation continue (ADORNI-BRACCESI et al., 1997 ; MASCIOCCO et al., 1989 ; MERLO et al., 1997), la période la plus intéressante pour analyser les modalités d'infiltration et le mouvement des eaux dans le sol est celle comprise entre septembre 1994 et août 1995. Cette période est en effet marquée par un épisode pluvieux exceptionnel $(300 \mathrm{~mm}$ de pluie en 48 heures), suivi d'une crue du fleuve Dora Baltea de fréquence centennale. $\AA$ cette occasion, la parcelle expérimentale fut complètement inondée, et donc « saturée ».

\section{2 - CARACTÉRISATION GÉOGRAPHIQUE ET GÉOLOGIQUE DU SITE}

Le centre ENEA-EUREX de Saluggia est située dans le secteur méridional de l'amphithéâtre morainique d'Ivrea, sur les berges du fleuve Dora Baltea et à proximité du canal Farini (figure 1). La parcelle, située au centre d'une surface non cultivée de $1000 \mathrm{~m}^{2}$, se développe sur les dépôts fluvio-glaciaires du Quaternaire, composés de graviers sableux avec intercalations de sables et de sables limoneux. Cette formation couvre les sédiments du Pléistocène, constitués d'une alternance de sables et de graviers avec des lentilles d'éléments plus fins (BONSIGNORE et al., 1969 ; BRAGA et al., 1989). Ces derniers reposent au-dessus des dépôts marins sableux de l'Astien et argileux du Plaisancien (Pliocène).

Les formations du Pléistocène et de l'Holocène renferment une nappe indifférenciée de forte puissance $(70$ à $80 \mathrm{~m}$ ) à une profondeur de 2 mètres environ (BORTOLAMI et al., 1987).

\section{3 - ACQUISITION DES DONNÉES ET MESURES}

Le site expérimental a été équipé de 6 bougies poreuses, de 6 tensiomètres à mercure et d'un tube d'accès pour la sonde à neutrons. Les lectures tensiométriques et humidimétriques ont eu lieu chaque semaine. Le prélèvement de la solution circulante a eu lieu chaque deux semaines. Pendant la période d'observation, les échantillons de sol ont été prélevés, deux fois, à la tarière, afin d'extraire, sous vide, la totalité de l'eau (ARAGUÀS-ARAGUÀS et al., 1995 ; JUSSERAND, 1980 ; WALKER et al., 1991) ; il s'agit non seulement de l'eau véhiculée dans la macroporosité et dans la microporosité, mais aussi de l'eau de rétention. 


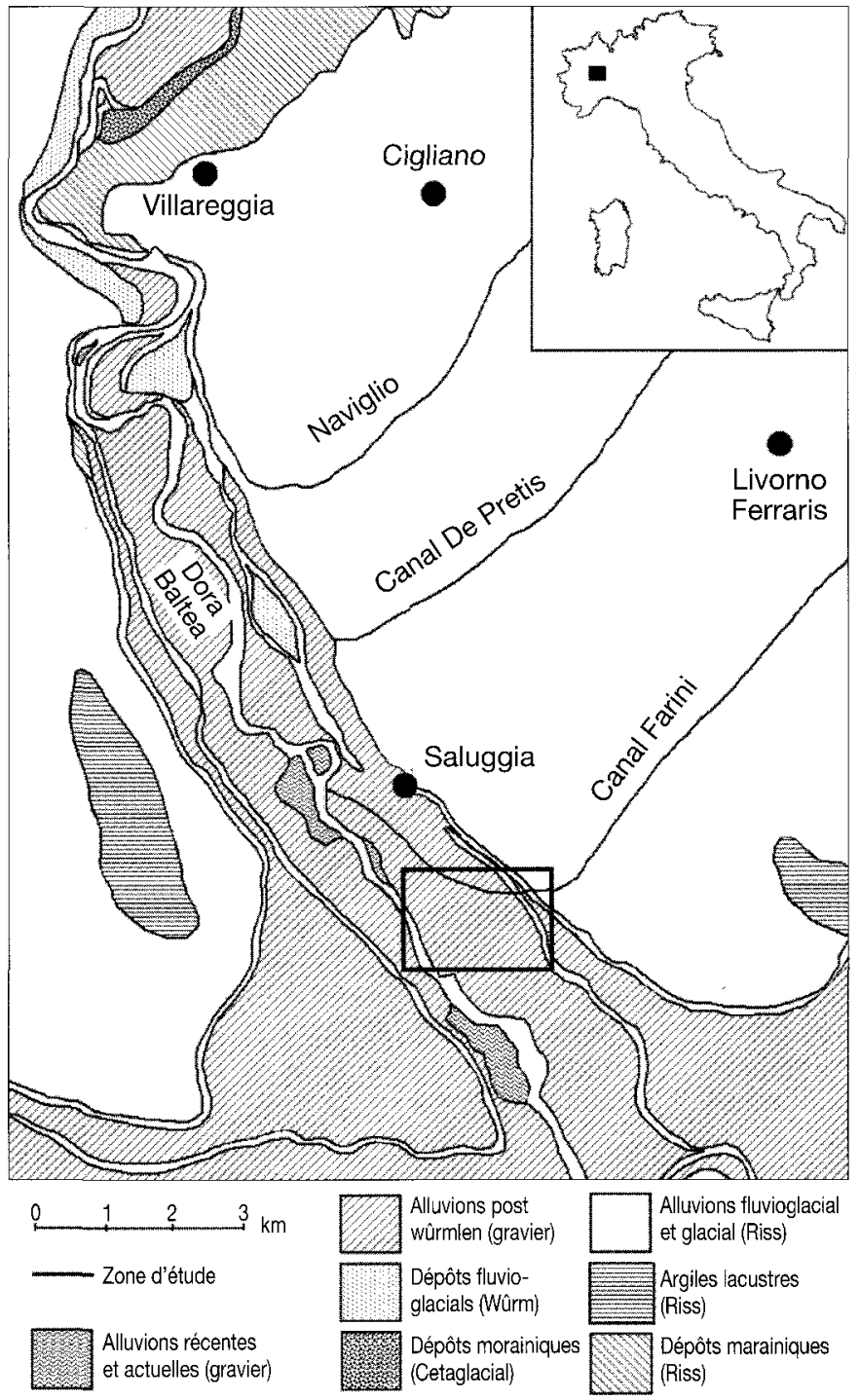

Figure 1 Schéma géologique de la zone de Saluggia (d'après BONSIGNORE et al., 1969).

Schematic geological map of the Saluggia district (taken from BONSIGNORE et al., 1969).

Les eaux de la nappe et du réseau de surface ont été aussi échantillonnées afin de définir les signaux chimiques et isotopiques d'entrée et d'évaluer les différents processus physicochimiques prenant place dans le sol.

Les échantillons ont été analysés au laboratoire d'Hydrogéologie et de Géochimie appliquée de l'Université de Turin, suivant les méthodes traditionnelles de l'absorption atomique (cations), de la chromatographie ionique (anions) et 
de la spectrométrie de masse (isotopes stables: ${ }^{2} \mathrm{H},{ }^{18} \mathrm{O}$ et ${ }^{13} \mathrm{C}$ ). Les compositions isotopiques des eaux et des calcites sont données par rapports aux étalons internationaux (Vienna-SMOW et Vienna-PDB) et sont exprimées en unité $\delta(\%)$ (GONFIANTINI, 1978). L'erreur analytique sur la mesure du rapport isotopique est de $\pm 1 \%$ o pour $\delta^{2} \mathrm{H}, \pm 0,1 \%$ o pour $\delta^{18} \mathrm{O}$ et $\pm 0,2 \%$ pour $\delta^{13} \mathrm{C}$.

Une station météorologique complète le site, fournissant les valeurs horaires de la température de l'air, de l'intensité de la radiation solaire, de l'humidité relative, de la direction et de l'intensité du vent et ainsi que celles des précipitations. Les eaux de pluie ont été récoltées dans un pluviomètre totalisateur à l'abri de l'évaporation, et prélevées à l'échelle mensuelle selon la méthode classique suggérée par l'IAEA (1983) ; l'échantillonnage a été complété par des prélèvements ponctuels correspondant à des événements particuliers.

\section{4 - RÉSULTATS}

\subsection{Sol}

Le sol présente une texture de type sableux, avec une faible présence d'argile. Dans la partie profonde du profil, les sables sont plus grossiers et la phase fine est moindre (tableau 1). Cette différence granulométrique contrôle la conductivité hydraulique du système, variable entre le sommet $\left(2 \cdot 10^{-4} \pm 1 \cdot 10^{-4} \mathrm{~ms}^{-1}\right)$ et la base du profil $\left(6 \cdot 10^{-4} \pm 2 \cdot 10^{-4} \mathrm{~ms}^{-1}\right)$ (MASCIOCCO et al., 1989).

Tableau 1 Granulométrie, composition chimique et paramètres de l'équation de Campbell pour le sol de Saluggia.

Table 1 Granulometry, chemical composition and Campbell's equation parameters for Saluggia soil.

\begin{tabular}{|c|c|c|c|c|c|c|}
\hline Profondeur (cm) & 25 & 50 & 75 & 100 & 125 & 150 \\
\hline Sable & 77 & 68 & 67 & 81 & 91 & 92 \\
\hline Limon & 20 & 29 & 31 & 17 & 8 & 8 \\
\hline Argile & 3 & 3 & 2 & 2 & 1 & 0 \\
\hline $\mathrm{pH}\left(\mathrm{en} \mathrm{H}_{2} \mathrm{O}\right)$ & 7,5 & 7,8 & 7,9 & 8,1 & 8 & 8,1 \\
\hline $\mathrm{pH}(\mathrm{en} \mathrm{KCl})$ & 7,5 & 7,3 & 7,2 & 7,7 & 7,8 & 8,0 \\
\hline Matière organique(\%) & 4,3 & 5,5 & 2,5 & 1,5 & 0,3 & 0,0 \\
\hline $\mathrm{CaCO}_{3}(\%)$ & 0,5 & 0,5 & 0,6 & 1,4 & 0,8 & 1,0 \\
\hline Capacité d'échange cationique & 10,02 & 6,75 & 8,95 & 9,39 & 8,11 & 7,81 \\
\hline $\begin{array}{c}\text { Paramètres de l'équation } \\
\text { de Campbell }\end{array}$ & \multicolumn{3}{|c|}{ Profondeur 0-75 cm } & \multicolumn{3}{|c|}{ Profondeur $75-150 \mathrm{~cm}$} \\
\hline a & \multirow{4}{*}{\multicolumn{3}{|c|}{$\begin{array}{r}-0,0120 \\
7,4030 \\
-0,0200 \\
0,3999\end{array}$}} & \multicolumn{3}{|c|}{$-0,1720$} \\
\hline $\mathrm{b}$ & & & & \multicolumn{3}{|c|}{2,6120} \\
\hline hc & & & & \multicolumn{3}{|c|}{$-0,1088$} \\
\hline$\theta \chi$ & & & & \multicolumn{3}{|c|}{0,3356} \\
\hline
\end{tabular}


La fraction fine a été étudiée par diffractométrie aux rayons $X$ sur toute la colonne. Les phases de la fraction fine sont représentées par de la kaolinite, accompagnée d'illite en quantité croissante avec la profondeur (MERLO et al., 1997).

La matière organique décroît de la couche superficielle du profil (jusqu'à $5,5 \%)$ aux couches inférieures $(0,6 \%)$. Le rapport $\mathrm{C} / \mathrm{N}$ est d'environ de 35 , suggérant ainsi une très lente décomposition de la matière organique (MASclocco et al., 1989).

Les carbonates sont toujours présents sur l'ensemble du profil ; du point de vue isotopique, ils témoignent de l'évolution temporelle des niveaux de la nappe (AFFEK et al., 1998), traduisant l'influence significative des acides organiques sur la composition isotopique du Carbone Totale Dissous des eaux de rétention dans la ZNS.

Pour obtenir les courbes de rétention relatives aux couches à texture type "sable " et "sable grossier ", les valeurs fournies par les tensiomètres ont été intégrées par des mesures à l'appareil de Richards lorsque les tensions dans le sol dépassaient le seuil limite d'utilisation des tensiomètres $(-70 \mathrm{kPa})$. Les tensions obtenues ont permis de calculer les paramètres de l'équation de CAMPBELL (1974) :

$$
\psi=a\left(\frac{\theta}{\theta_{\text {sat }}}\right)^{-b}
$$

où $\psi$ est la tension ( $\mathrm{kPa}$ ), $\theta$ l'humidité actuelle du sol, $\psi_{\text {sat }}$ l'humidité à la saturation, a la valeur de la pression de l'air $(\mathrm{kPa})$ et $\mathrm{b}$ un coefficient empirique (sans dimension).

\subsection{Eaux météoriques}

Les variations de la composition chimique des eaux mettent bien en évidence les effets conjugués des mouvements sur l'Europe des masses d'air en provenance de l'océan Atlantique et de la Méditerranée. En effet, les précipitations d'été, liées à la condensation de la vapeur d'origine locale, sont moins chargées en ions que celles d'origine méditerranéenne ou atlantique (DRAY et al., 1998 ; NOVEL et al., 1996) ; celles-ci entraînent les signaux des embruns marins et de la pollution continentale.

Les isotopes stables de l'eau marquent la cadence saisonnière des eaux météoriques, et leurs différentes origines. La moyenne annuelle pondérée en $\delta^{18} \mathrm{O}$ de l'eau de pluie à Saluggia est de $-7,10 \% \pm 0,66$; la droite météorique locale calculée :

$$
\delta^{2} H=8,02 \delta^{18} O+10,5
$$

\subsection{Eaux de surface}

Durant l'année d'observation, les eaux de la Dora Baltea et du canal Farini montrent une composition isotopique évoluant d'une façon similaire, avec une valeur moyenne en $\delta^{18} \mathrm{O}$ de $-10,71 \% \pm 0,67$ pour la rivière et de $-10,62 \% \pm 0,73$ pour le canal (figure 2 ). Les valeurs plus négatives sont toujours affichées à la fin du printemps, à la suite du passage des eaux de fonte de neige en provenance du cœur de la chaîne alpine. Toutefois, l'oscillation 


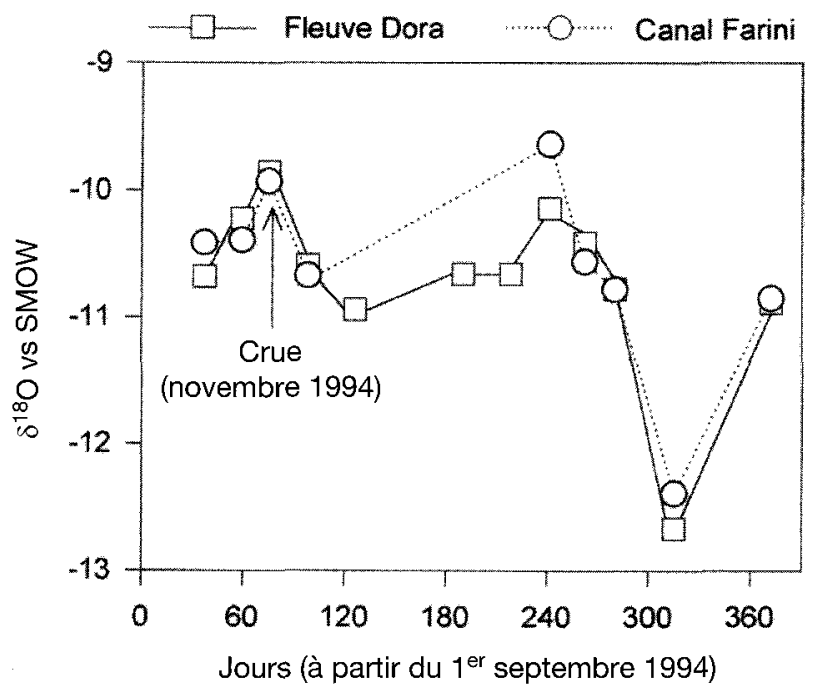

Figure 2 Variation avec le temps de la composition isotopique $\left(\delta^{18} \mathrm{O}\right)$ des eaux de la Dora Baltea et du canal Farini.

Temporal variations of Oxygen-18 content in surface waters (Dora Baltea and Farini Canal).

saisonnière est atténuée par le fonctionnement de nombreuses centrales hydroélectriques situées dans le val d'Aoste qui homogénéisent les signaux météoriques (NOVEL et al., 1996).

\subsection{Eaux de la nappe phréatique}

Les eaux de la nappe phréatique sont contrôlées, du point de vue hydrodynamique, par la Dora Baltea. Pour la période d'observation, elles présentent une valeur moyenne en $\delta^{18} \mathrm{O}$ de $-8,09 \% \pm 0,80$, valeur plus enrichie par rapport à la composition isotopique du fleuve. L'enrichissement isotopique est la conséquence de la forte évaporation à laquelle sont soumises les eaux dans les rizières : elles sont mobilisées, par la suite, dans le réseau d'irrigation et donnent une sensible contribution latérale à la recharge de la nappe. II faut, en outre, souligner qu'à la suite de la crue du 5 novembre 1994, et donc à la complète saturation du sol par les eaux de surface, les différences de composition isotopique entre les eaux de la nappe phréatique et celles de la Dora disparaissent. Toutefois, après quelques semaines, elles retrouvent chacune leurs caractéristiques propres, montrant de nouveau un écart significatif.

\subsection{Eaux d'infiltration}

Les valeurs isotopiques moyennes des échantillons d'eau obtenue par distillation sous vide ou extraite par bougies poreuses (JUSSERAND, 1980) présentent toujours deux courbes évoluant de manière similaire (figure 3), avec cependant un écart constant entre elles. Dans le premier cas, l'eau présente une valeur moyenne en $\delta^{18} \mathrm{O}$ de $-10,33 \% \pm 1,13$, valeur comparable à celle de l'eau de la Dora ; en revanche, l'eau des bougies poreuses affiche une 

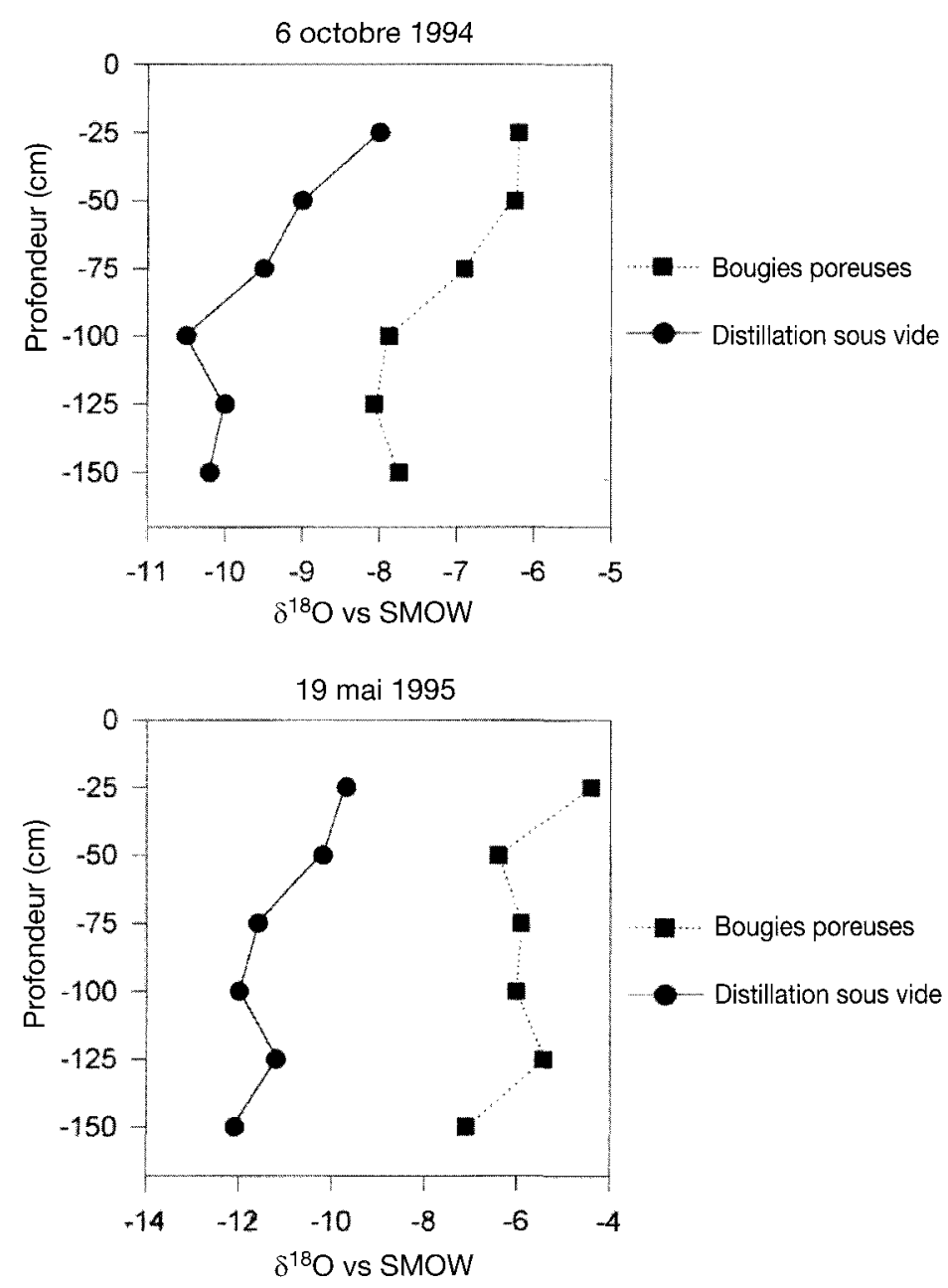

Figure 3 Profils de la composition isotopique des eaux extraites du sol par distillation sous vide et par bougies poreuses le 6 octobre 1994 et le 19 mai 1995.

Oxygen-18 content of water extracted from the soil under vacuum conditions and with porous cups (6 October 1994, and 19 May 1995).

valeur moyenne du $\delta^{18} \mathrm{O}$ de $-6,53 \% \pm 0,98$, proche de la moyenne annuelle des précipitations locales. L'écart entre la composition isotopique des eaux extraites par ces deux méthodes marque la différence entre la circulation de l'eau à l'intérieur de l'ensemble des vides (macroporosité et microporosité, circulation lente) et celle à l'intérieur de la macroporosité (circulation rapide). En effet, l'eau contenue dans la microporosité, véhiculée plus lentement dans le terrains, est préalablement soumise aux phénomènes de dispersion homogénéisant les signaux météoriques descendantes avec les signaux remontant de la nappe par capillarité. Dans le cas de l'eau contenue dans la macroporosité, les composantes diffusives et convectives peuvent être égales ; la diffusion a 
lieu à cause de la dimension plus grande des vides et de leur interconnexion qui, en même temps, facilitent le phénomène advectif (ALLISON, 1995). Par conséquent, dans la macroporosité les derniers signaux météoriques peuvent s'homogénéiser seulement avec les signaux immédiatement précédents et, éventuellement, leur effet est limité aux signaux suivants. Ainsi, la masse avancerait d'une façon impulsionnelle, comme le confirmerait la variation interannuelle de la composition chimique et isotopique de l'eau extraite par les bougies poreuses (ADORNI-BRACCESI et al., 1997 ; MERLO et al., 1997).

Les eaux interstitielles extraites à l'aide des bougies poreuses se situent, dans le diagramme classique $\delta^{2} \mathrm{H}$ vs $\delta^{18} \mathrm{O}$, le long d'une droite dont la pente est de 4,9 . Le point d'intersection avec la droite météorique locale est de $\delta^{2} \mathrm{H}=-76,7 \%$ et $\delta^{18} \mathrm{O}=-10,87 \%$ : ce point correspond à la moyenne interannuelle des eaux de la Dora (figure 4).

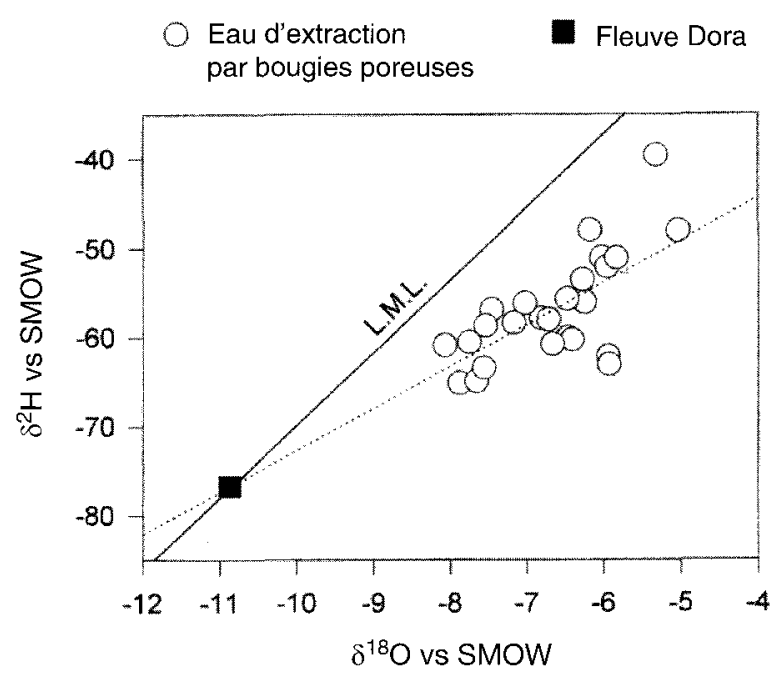

Figure 4 Composition isotopique des eaux d'extraction par bougies poreuses.

Isotopic composition of waters extracted with porous cups (LMWL equation).

Les valeurs de la conductivité électrique des eaux d'infiltration varient significativement en fonction de la profondeur et de la période considérée (figure 5). Une variation saisonnière se manifeste clairement, comme en témoignent les valeurs plus basses observées en hiver en raison de la dilution par les précipitations d'une part, et de l'activité biologique réduite, d'autre part. La concentration ionique augmente au printemps et atteint un maximum en été à la suite de l'accroissement combiné de l'intense activité biologique et de l'évaporation. Enfin, à l'automne, la remobilisation, par les pluies, de l'eau résiduelle de l'été et des composés précipités ou piégés dans les interstices de la ZNS, conduit à une forte augmentation de la charge ionique des eaux d'infiltration. Cette remobilisation intéresse essentiellement les nitrates formés par la matière organique à la fin du cycle végétatif. 
Automne

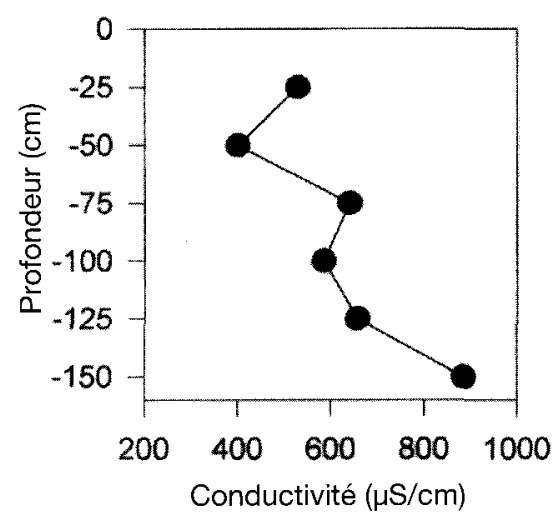

Printemps

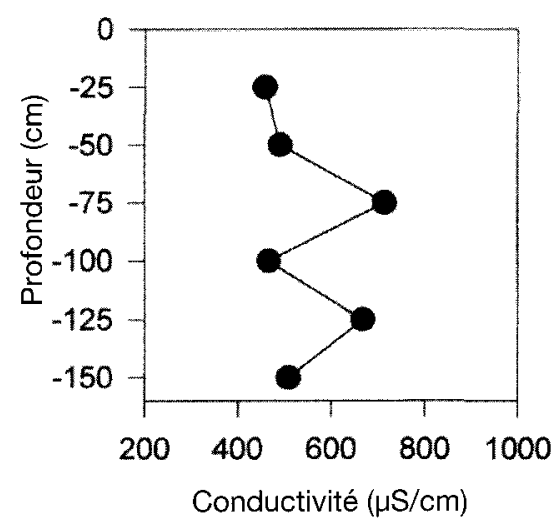

Hiver

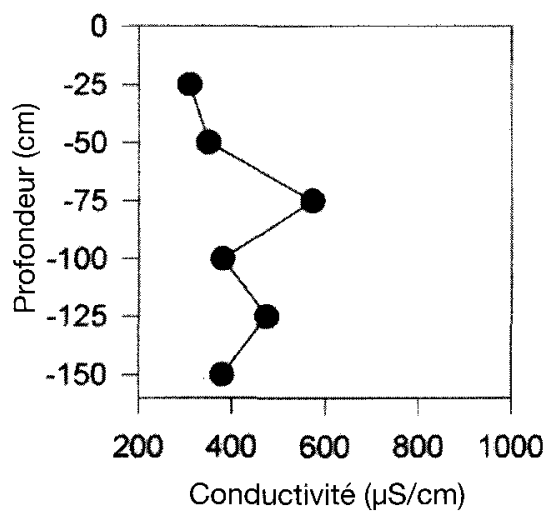

Été

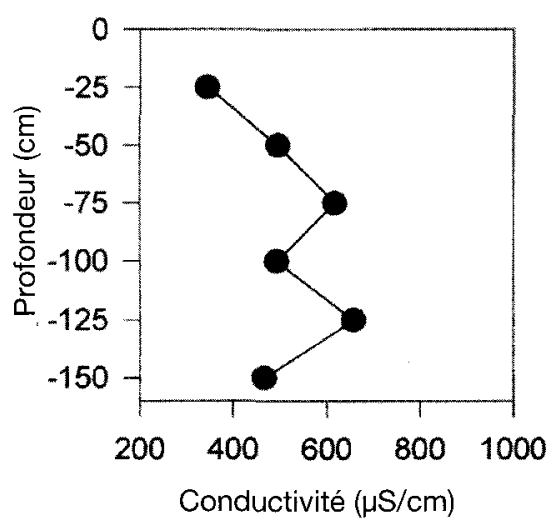

Figure 5 Profils de la conductivité électrique (en $\mu \mathrm{S} / \mathrm{cm}$ ) des eaux d'extraction. Electrical conductivity profiles (in $\mu \mathrm{S} / \mathrm{cm}$ ) for extracted pore waters.

\section{5 - ÉVALUATION DU BILAN HYDRIQUE}

\subsection{Méthodes physiques : bilan hydrique de masse par modèle monocouche}

Le bilan hydrique du sol a été déterminé en considérant le sol comme un unique réservoir monocouche sans tenir compte des niveaux à granulométrie différente et faisant appel à la formule de Turc (1961) pour l'estimation de l'évapotranspiration. La Réserve Facilement Utilisable (RFU) calculée sur la base des données mesurées de tension et humidité, affiche une valeur de $84,3 \mathrm{~mm}$. La recharge de la nappe pour la période septembre 1994-août 1995 est de $143 \mathrm{~mm}$ d'eau, soit $19 \%$ du total des précipitations ; l'excès en eau est apparemment limité à l'automne et à une partie de l'été, alors que le déficit hydrique est observé dans les autres mois (tableau 2). 
Tableau 2 Recharge évaluée par la méthode du bilan hydrique pour la période d'observation.

Table 2 Recharge evaluated with the water mass balance method for the study period.

\begin{tabular}{|c|c|c|c|c|c|c|c|c|c|c|c|c|c|}
\hline & Sept & Oct & Nov & Déc & Janv & Fév & Mars & Avr & Mai & Juin & Jul & Août & Tot (mm) \\
\hline Pluie & 211,7 & 46,7 & 194,8 & 1,1 & 1,1 & 3,8 & 1,0 & 2,9 & 5,5 & 76,7 & 26,2 & 179,6 & 751,1 \\
\hline ETP & 58,9 & 20,0 & 14,1 & 6,0 & 0,1 & 16,5 & 38,8 & 62,1 & 87,1 & 100,9 & 115,5 & 87,9 & 607,9 \\
\hline Pluie-ETP & 152,8 & 26,7 & 180,7 & $-4,9$ & 1,0 & $-12,7$ & $-37,8$ & $-59,2$ & $-81,6$ & $-24,2$ & $-89,3$ & 91,7 & 143,2 \\
\hline RFU & 84,3 & 84,3 & 84,3 & 79,4 & 80,4 & 67,7 & 29,9 & 0,0 & 0,0 & 0,0 & 0,0 & 84,1 & \\
\hline Variation & 0,0 & 0,0 & 0,0 & $-4,9$ & 1,0 & $-12,7$ & $-37,8$ & $-29,9$ & 0,0 & 0,0 & 0,0 & 91,7 & \\
\hline ETR & 58,9 & 20,0 & 14,1 & 6,0 & 0,1 & 16,5 & 38,8 & 32,2 & 5,5 & 76,7 & 26,2 & 87,9 & 382,9 \\
\hline Déficit & 0,0 & 0,0 & 0,0 & 0,0 & 0,0 & 0,0 & 0,0 & 29,3 & 81,6 & 24,2 & 89,3 & 0,0 & 224,4 \\
\hline Recharge & 152,8 & 26,7 & 180,7 & 0,0 & 0,0 & 0,0 & 0,0 & $-29,3$ & $-81,6$ & $-24,2$ & $-89,3$ & 7,6 & 143,4 \\
\hline
\end{tabular}


Tableau 3 Mesures physiques du sol; données de conductivité, chlorure, nitrate et composition isotopique des eaux de pores.

Table 3 Physical soil measurements; conductivity, $\mathrm{Cl}$ and $\mathrm{NO}_{3}^{-}$content and isotopic composition of pore water.

\begin{tabular}{|l|c|c|c|c|}
\cline { 2 - 5 } \multicolumn{1}{c|}{} & \multicolumn{4}{c|}{ Conductivité $(\boldsymbol{\mu S} / \mathbf{c m})$} \\
\cline { 2 - 5 } \multicolumn{1}{c|}{} & Min & Max & Moyenne & Écart type \\
\hline Automne & 348 & 993 & 655 & 178 \\
Hiver & 287 & 573 & 379 & 79 \\
Printemps & 379 & 893 & 532 & 115 \\
Été & 228 & 789 & 553 & 145 \\
Sept 1994-A0ût 1995 & $\mathbf{2 2 8}$ & $\mathbf{9 9 3}$ & $\mathbf{5 4 2}$ & $\mathbf{1 5 9}$ \\
\hline
\end{tabular}

\begin{tabular}{|c|c|c|c|}
\hline \multicolumn{4}{|c|}{$\mathbf{C l}^{-}$(mg/l) } \\
\hline Min & Max & Moyenne & Écart type \\
\hline 1,1 & 86,0 & 23,5 & 21,2 \\
1,1 & 12,3 & 1,9 & 0,6 \\
1,2 & 18,5 & 5,2 & 5,9 \\
1,3 & 28,2 & 6,5 & 9,2 \\
$\mathbf{1 , 1}$ & $\mathbf{8 6 , 0}$ & $\mathbf{9 , 3}$ & $\mathbf{1 4 , 3}$ \\
\hline
\end{tabular}

\begin{tabular}{|c|c|c|c|}
\hline \multicolumn{4}{|c|}{$\mathrm{NO}_{\mathbf{3}}^{-}(\mathbf{m g} / \mathbf{l})$} \\
\hline Min & Max & Moyenne & Écart type \\
\hline 0,0 & 87,9 & 15,0 & 24,3 \\
0,0 & 65,4 & 11,4 & 18,4 \\
0,0 & 212,1 & 25,3 & 52,1 \\
0,0 & 84,4 & 11,7 & 24,5 \\
$\mathbf{0 , 0}$ & $\mathbf{2 1 2 , 1}$ & $\mathbf{1 6 , 6}$ & $\mathbf{3 5 , 1}$ \\
\hline
\end{tabular}

\begin{tabular}{|l|c|c|c|c|}
\cline { 2 - 5 } \multicolumn{1}{c|}{} & \multicolumn{4}{c|}{$\delta^{18}$ O vs SMOW } \\
\cline { 2 - 5 } \multicolumn{1}{c|}{} & Min & Max & Moyenne & Écart type \\
\hline Automne & $-9,09$ & $-5,32$ & $-7,17$ & 1,12 \\
Hiver & $-7,66$ & $-5,13$ & $-6,76$ & 0,81 \\
Été & $-8,07$ & $-4,14$ & $-6,34$ & 0,96 \\
Sept 1994-Août 1995 & $-7,90$ & $-3,25$ & $-6,58$ & 1,38 \\
& $-9,09$ & $-3,25$ & $-6,67$ & 1,15 \\
\hline
\end{tabular}

\begin{tabular}{|c|c|c|c|}
\hline \multicolumn{4}{|c|}{$\psi(\mathbf{k P a})$} \\
\hline Min & Max & Moyenne & Écart type \\
\hline$-10,7$ & 0,0 & $-5,38$ & 13,8 \\
$-43,6$ & $-0,1$ & $-31,4$ & 70,3 \\
$-119,8$ & $-0,1$ & $-45,3$ & 136,8 \\
$-55,9$ & $-0,1$ & $-47,7$ & 88,6 \\
$-50,4$ & 0,0 & $-47,7$ & 88,1 \\
\hline
\end{tabular}

\begin{tabular}{|c|c|c|c|}
\hline \multicolumn{3}{|c|}{$\theta$} \\
\hline Min & Max & Moyenne & Écart type \\
\hline 0,06 & 0,34 & 0,20 & 0,06 \\
0,10 & 0,25 & 0,16 & 0,05 \\
0,09 & 0,20 & 0,13 & 0,03 \\
0,09 & 0,29 & 0,15 & 0,05 \\
0,06 & 0,34 & 0,16 & 0,05 \\
\hline
\end{tabular}




\subsection{Méthodes physiques : bilan hydrique de masse par modèle multicouche}

Le bilan hydrique de chaque couche a été calculé par l'équation du flux hydrique en régime transitoire (équation de Richards) : cette équation généralise la loi de Darcy aux conditions de non saturation et fait appel à la relation existante entre tension et saturation, et entre celle-ci et la conductivité hydraulique.

Le mouvement de l'eau à travers le sol a été évaluée à l'aide du programme de modélisation LEACHM (HUTSON et WAGENET, 1992), programme qui utilise les données pluviométriques et d'évapotranspiration potentielle locales à l'échelle journalière (ALLEN et al., 1998), les données de tension et humidité (tableau 3) et qui prend en compte l'évolution de la couverture végétale et le développement des racines. À la suite de la crue de la Dora Baltea, la période de submersion a été modélisée en considérant la surface de la parcelle sous un niveau d'eau constant.

Les mouvements de l'eau sont calculés pour un temps égal ou inférieur à 0,1 jour ; toutefois les données sont reportées en tant que moyennes hebdomadaires. Le modèle a montré une très bonne concordance avec les données expérimentales de tension et d'humidité du sol. L'indice d'efficience du modèle (LOAGUE et GREEN, 1991), qui doit être le plus proche possible de 1, est de 0,81 . L'infiltration efficace a été estimée à $224 \mathrm{~mm}$ (29\% du total des précipitations) (figure 6).

Par ce modèle, il est possible de définir la position du Plan de Flux Nul (PFN) dans le temps et en profondeur avec une résolution supérieure à celle des tensiomètres. En effet il arrive que le PFN se situe au-dessus de la profondeur d'observation maximale, mais seulement pendant les mois caractérisés par d'abondantes précipitations (automne) ou par une évapotranspiration faible (hiver). Durant le printemps, la migration vers le bas du PFN est observée en relation avec les faibles précipitations et la forte évapotranspiration. La descente est encore plus évidente en été, quand le PFN se situe au-dessous de la profondeur maximale mesurée et, théoriquement, aussi en pleine frange capillaire ; dans ce cas, il s'agit d'une perte par évaporation directe de la nappe.

\subsection{Méthode isotopique}

Les pertes de la nappe phréatique par évaporation directe ont été quantifiées en considérant la composition isotopique des eaux de la nappe et des eaux extraites par les bougies poreuses. L'évaporation serait d'autant plus importante que les eaux sont prélevées près de la surface et que la période entre deux précipitations est longue. Par ailleurs, nombreuses sont les études qui ont montré un enrichissement isotopique affectant les eaux souterraines quand le niveau piézométrique se rapproche de la surface (ALLISON, 1995 ; IAEA, 1983).

En tenant compte du contenu hydrique à différentes profondeurs, de la tortuosité (ALLISON 1995 ; FONTES et EDMUNDS, 1989), et en utilisant comme valeur de la diffusivité $D_{180}=1,45 \cdot 10^{-9} \mathrm{~m}^{2} \mathrm{~s}^{-1}$ (BARNES et ALLISON, 1983), l'évaporation directe annuelle de la nappe a été évaluée en $3 \cdot 10^{-3} \mathrm{~m}^{3} \mathrm{~m}^{-2} \mathrm{a}^{-1}$. 


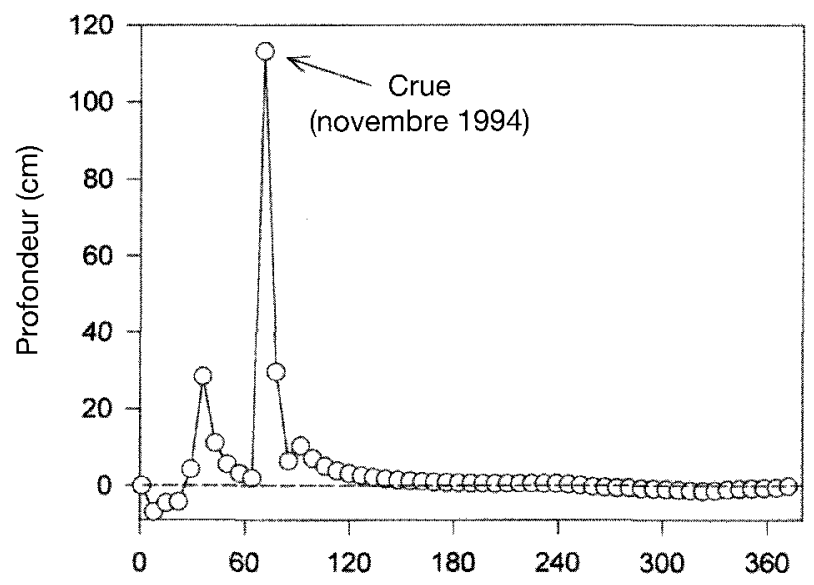

Jours (à partir du $1^{\text {er }}$ septembre 1994)

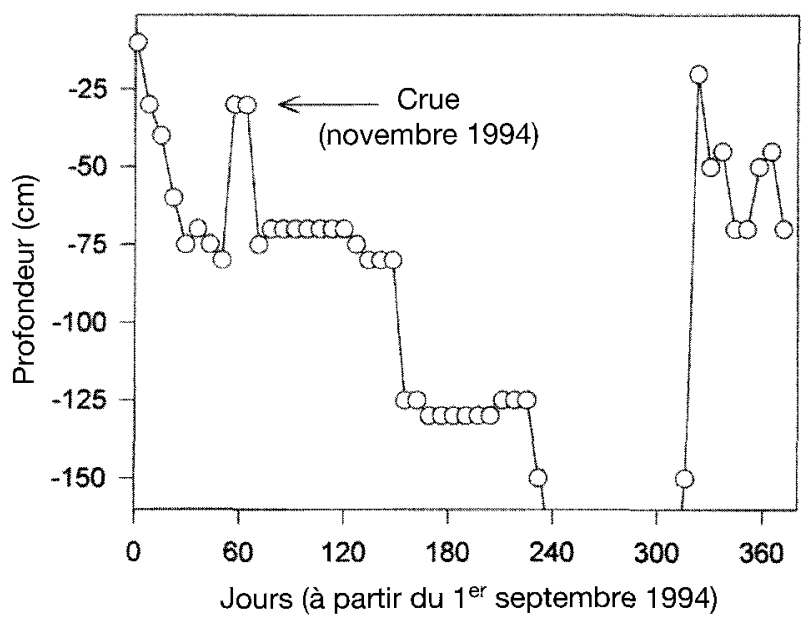

Figure 6 Recharge évaluée par la méthode du bilan hydrique et variation dans le temps de la profondeur du Plan de Flux Nul.

Recharge rate evaluated by the water balance method, and temporal variation in depth of the Zero Flux Plane.

\subsection{Méthode géochimique}

Le principe de cette méthode repose sur la connaissance du rapport de concentration des ions conservatifs dans l'eau des précipitations et dans l'eau du sol ; ce rapport est directement imputable à la reprise évaporatoire affectant uniquement la molécule de l'eau. L'ion chlorure est considéré comme l'ion le plus approprié pour telles mesures, du moment qu'il montre une absorption transcurable vis-à-vis des sols. En plus, en présence d'une conductivité hydraulique élevée, le transport est contrôlé par la vitesse advective moyenne. Du moment que le profil des chlorures dans le sol peut être considéré, en 
général, stationnaire pendant la période considérée, le flux en eau (q) peut être calculé par l'équation suivante (SHARMA, 1988) :

$$
q=\frac{1}{C_{z}}\left(J+D \theta \frac{\partial C_{z}}{\partial z}\right)
$$

où $\mathrm{Cz}$ est la concentration moyenne annuelle en chlorures dans les eaux du sol, $J$ le flux vertical unidimensionnel, $D$ le coefficient de diffusion du soluté, $\theta$ la saturation en eau du sol et $z$ la profondeur.

Du moment que la composante diffusive peut être ignorée, la formule (3) devient :

$$
q=\frac{J}{C_{z}}
$$

Si on assume que les chlorures proviennent uniquement des précipitations, l'expression du flux annuelle (J) peut s'écrire :

$$
J=C_{\rho} \times P
$$

où $\mathrm{Cp}$ indique la concentration des chlorures dans l'eau de pluie et $\mathrm{P}$ la hauteur moyenne annuelle des précipitations.

On en déduit alors la recharge $(R)$ :

$$
R=\frac{C_{p} \times P}{C_{z}}
$$

Cette méthode permet d'évaluer la recharge à Saluggia, à environ $90 \mathrm{~mm}$, soit $12 \%$ du total des précipitations annuelles.

\section{6 - INTERACTION EAU-SOL}

Les interactions eau-sol ont été prises en considération, afin de suivre les mécanismes intervenant lors de l'installation des processus de percolation et de décrire l'évolution biogéochimique de la matière organique dans le sol. La question qui se pose toujours dans ces études concerne la représentativité chimique de l'eau extraite par bougies par rapport à l'eau de la solution dans le sol. La chimie de l'eau dépend des nombreux facteurs : l'état de saturation du sol, la succion appliquée pour l'extraction, la nature du sol et ses propriétés. Toutefois, le problème de la macroporosité et de la microporosité reste sousjacent pour expliquer les différences de composition chimique entre l'eau extraite par succion et l'eau restante dans le sédiment : des variations de $\mathrm{pH}$ de 2 à 3 unités ont été régulièrement observée (MERLO et al., 1997), en accord avec les données de la littérature (DAHLGREN, 1993 ; GROSSMANN et UDLUFT, 1991 ; HANSEN et HARRIS, 1975). Toutefois, comme il existe des échanges gazeux entre l'échantillon et l'atmosphère qui règne dans le système d'extraction, mais même dans la zone soumise à extraction, il va de soi que l'alcalinité 
et le $\delta^{13} \mathrm{C}$ de telles solutions extraites par bougies poreuses peuvent indiquer correctement la minéralisation du carbone dans la zone non saturée.

L'indice de saturation vis-à-vis de la calcite calculé à l'aide du programme PCWATEQ (TRUESDELL et JONES, 1974), montre que les eaux échantillonnées avec les bougies poreuses se situent normalement au-dessus de l'équilibre thermodynamique avec la phase solide (figure 7).

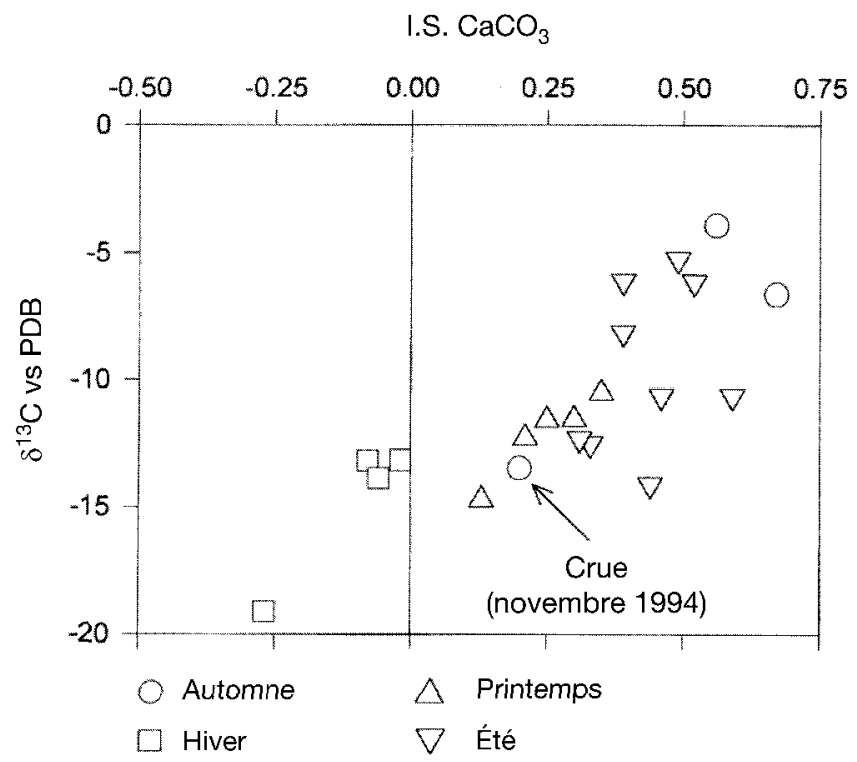

Figure 7 Variation de la composition isotopique $\left(\delta^{13} \mathrm{C}\right)$ du Carbone Total Dissous en fonction de l'indice de saturation par rapport à la calcite, dans les eaux d'extraction par bougies poreuses.

Variation in the $\delta^{13} \mathrm{C}$ isotope content of the Total Dissolved Carbon as a function of the Calcite Saturation Index in pore waters.

Pendant l'hiver, la percolation vers la nappe de l'eau météorique caractérisée par un $\mathrm{pH}$ légèrement acide, déplace l'équilibre vers des valeurs de sous-saturation. Au contraire, durant les mois à faibles précipitations, l'évaporation intense conduit à une sursaturation qui favorise la précipitation des carbonates. Toutefois, le point correspondant à la crue centennale de la Dora (novembre 1994) fait exception. L'excès en eau provoque le lessivage dans le sol d'une quantité non négligeable de $\mathrm{CO}_{2}$ biogénique : la solution infiltrante est donc plus acide et les eaux sont déplacées vers l'équilibre. Ce cycle, à l'échelle annuelle, est semblable aux cycles de la précipitation de la calcite observée dans les lacs de profondeur réduite où la calcite se forme à la suite de la hausse de température qui augmente la fugacité du $\mathrm{CO}_{2}$ dissous (CERLING, 1984 ; WOOD et PETRAITIS, 1984.) Le niveau de calcite néoformée, surtout quand l'hiver qui suit est sec, reste le témoignage de la situation climatique précédente. 


\section{7 - CONCLUSION}

L'application des différentes méthodes physiques et chimiques pour évaluer l'évapotranspiration et l'infiltration dans le site de Saluggia pour la période d'observation (septembre 1994-août 1995), a fourni des résultats sensiblement différents.

L'utilisation d'un programme de modélisation (LEACHM), ayant simulé l'évolution de la tension et de l'humidité dans le sol est probablement l'outil le plus performant parmi ceux qui ont été utilisés. Cette méthode a permis d'évaluer la recharge, en prenant en compte les caractéristiques hydrodynamiques, physicochimiques et végétatives du sol. Les résultats obtenus donnent une infiltration efficace de l'ordre de $224 \mathrm{~mm}$, soit $29 \%$ des précipitations.

La tensiométrie et l'humidimétrie du sol, qui donnent la répartition de la teneur en eau le long du profil, ont permis d'étudier la position du PFN, qui se situe près de la surface pendant les mois à fortes précipitations (automne) ou à évapotranspiration faible (hiver). Au contraire, durant le printemps, le PFN migre vers le bas. Cette migration est encore plus évidente en été, alors que le PFN se situe en dessous de la profondeur maximale de la zone étudiée. Ce fait engendre un flux évaporatoire direct (en phase liquide et en phase vapeur) de la nappe vers l'atmosphère, et par conséquent des pertes de la ressource souterraine.

L'humidimétrie, elle-même ou en tant que base d'un modèle hydrologique, est donc un outil plus précis, mais demande des mesures continues et non ponctuelles, ou bien des données indirectes tels que les enregistrements météorologiques, les paramètres du sol et la connaissance détaillée de la couverture végétale.

Le bilan hydrique simplifié (monocouche) a permis d'évaluer une alimentation de la nappe de $143 \mathrm{~mm}$ (19\% du total des précipitations). Cette valeur est sous-estimée, car les paramètres considérés sont empiriques et ne tiennent pas compte des caractéristiques du sol, de la couverture végétale, et de la proximité de la nappe par rapport à la surface et donc du fait que l'eau infiltrée n'atteint guère un niveau à transpiration nulle. En effet, au moment des précipitations, l'eau percole rapidement vers le bas, mouvement facilité par l'humidité. Elle pourrait donc être véhiculée à l'intérieur de zones à faible ou à moyenne conductivité hydraulique. L'excédent hydrique est apparemment limité à l'automne, et à une partie de l'été (quand l'irrigation atteint son maximum) alors que le déficit hydrique apparaît pour les autres mois de l'année en fonction de l'activité biologique.

L'utilisation de l'outil chimique a permis d'estimer l'infiltration efficace de l'ordre de $12 \%$ des précipitations annuelles. Toutefois, cette méthode doit être appliquée avec circonspection, car la teneur des chlorures issus de la décomposition de la matière organique dans le sol est mal connue (DAVISSON et al., 1994 ; FLEISCHER et al., 1977). II faut souligner aussi que, dans l'application de cette méthode, l'hypothèse d'un état stationnaire est partiellement respecté, comme l'humidimétrie le montre.

Enfin, l'étude isotopique des eaux extraites du sol a permis de définir les pertes de la nappe et la dynamique de l'infiltration dans les différentes domaines 
du flux : la macroporosité, la microporosité et les eaux de rétention. Seulement pendant l'hiver, l'infiltration semble être de type " piston flow " dispersif (ARANYOSSY et al., 1995), selon un flux dirigé vers le bas, où la composante convective dans la microporosité est prédominante sur la composante diffusive ayant lieu, de préférence, dans la macroporosité. Au contraire, dès que les précipitations du printemps arrivent, jointes à une irrigation intense et à la température de l'air qui augmente, l'activité végétative commence, et donc, la composante diffusive devenue prioritaire, traduit un flux alternant la direction vers la surface au mouvement selon la gravité.

Pour expliquer la quantité et aussi la qualité des ressources souterraines dans cette région, il faut faire appel à la recharge latérale ; celle-ci étant plus importante que l'infiltration verticale; elle a lieu là où les grandes vallées alpines débouchent sur la plaine et où elle est favorisée par le réseau de canaux d'irrigation (ARANYOSSY et al., 1995).

\section{REMERCIEMENTS}

Les auteurs remercient G. Carbotta, G. Coppo et R. Cossio (Université de Turin) qui ont assuré le travail sur le terrain et au laboratoire. Ils se sentent aussi obligés vers F. Troiani, E. Pirastru et V. Calì du centre ENEA-EUREX de Saluggia pour l'aide logistique toujours apportée et vers C. Jusserand (CRG, Thonon-les-Bains) pour ses précieux conseils techniques et scientifiques.

\section{RÉFÉRENCES BIBLIOGRAPHIQUES}

ADORNI-BRACCESI A., CARBOTTA G., COPPO G., COSSIO R., MERLO S., SACCHI E., ZUPPI G.M., 1997. L'apporto della chimica e della fisica allo studio della Zona Non Satura : limiti tecnici, scientifici e metodologici. Quad. Geol. Appl., 3 (2), 137-146.

AFFEK H.P., RONEN D., YAKIR D., 1998. Production of $\mathrm{CO}_{2}$ in the capillary fringe of a deep phreatic aquifer. Water Resour. Res., 34, 989-996.

ALLEN R.G., PEREIRA L.S., RAES D., SMITH M., 1998. Irrigation and drainage. $\mathrm{FAO}$, Rapport scientifique $n^{\circ} 56,220 \mathrm{p}$.

ALLISON G.B., 1988. A review of some of the physical, chemical and isotopic techniques available for estimating groundwater recharge. In: Estimation of natural groundwater recharge. SIMMERS I. (Ed.), pp. 49-72.

ALLISON G.B., 1995. Stable isotopes in soil and water studies. Comptes rendus Symposium international à la mémoire de Jean Charles Fontes sur l'hydrologie et la géochimie isotopique. Paris, France, 1-2 June, 22-38.

ARAGUÀS-ARAGUÀS L., ROZANSKI K., GONFIANTINI R., LOUVAT D., 1995. Isotope effects accompanying vacuum extraction of soil water for stable isotope analyses. J. Hydrol., 168, 159-171.

ARANYOSSY J.F., NJITCHOUA R., ZUPPI G.M., 1995. L'apport des isotopes de l'environnement à l'étude de la recharge et de la dynamique des aquifères. Comptes rendus Symposium inter- 
national à la mémoire de Jean Charles Fontes sur l'hydrologie et la géochimie isotopique. Paris, France, 1-2 June, 3954.

BARNES C.J., ALLISON G.B., 1983. The distribution of deuterium and oxygen-18 in dry soils: I. Theory. J. Hydrol., 60, 141-156.

BONSIGNORE G., BORTOLAMI G.C., ELTER G., MONTRASIO A., PETRUCCI F., RAGNI U., SACCHI R., STURANI C., ZANELLA E., 1969. Note illustrative alla Carta Geologica d'Italia alla scala 1:100.000. Foglio 56-Torino e Foglio 57Vercelli. Poligrafica e Cartevalori (Ed.), Ercolano (NA), Italia, $96 \mathrm{p}$.

BORTOLAMI G.C., DI MOLFETTA A., MERLO G., 1987. Studio idrogeologico per il potenziamento della centrale idropotabile di Volpiano (TO). Geologia Tecnica, $14 \mathrm{p}$.

BRAGA G., CARABELlI E., CERVO A., COLOMBETTI A., D'OFFIZI S., FRANCAVILLA F., GASPERI G., PARMIGIANI L., PELLEGRINI M., ZAULI M., ZUPPI G.M., 1989. Indagini idrogeologiche nella pianura padana. Le aree del Piemonte (PO1 e PO2) e della Lombardia (Viadana e S. Benedetto). ENEL, Roma, Italia, Rapport scientifique : Contributi di preminente interesse scientifico agli studi di localizzazione di impianti nucleari in Piemonte e Lombardia, 550-671.

CAMPBELL G., 1974. A simple method for determining unsatured conductivity from moisture retention data. Soil Sci., 117, 311-314.

CERLING T.E., 1984. The stable isotopic composition of modern soil carbonate and its relationship to climate. Earth Planet. Sci. Lett., 71, 229-240.

CNR-IRSA, 1979. Indagine sulle falde acquifere profonde della pianura padana : lineamenti idrogeologici. Quaderni IRSA, 28, $135 \mathrm{p}$.

CNR-IRSA, 1981. Indagine sulle falde acquifere profonde della pianura padana : contributi tematici per la conoscenza dell'idrogeologia padana. Quaderni IRSA, 51 (II), $150 \mathrm{p}$.

DAHLGREN R.A., 1993. Comparison of soil solution extraction procedures. Effect on solute chemistry. Commun. Soil Sci. Plant Anal., 24 (15-16), 1783-1794.
DARLING W.G., BATH A.H., 1988. A stable isotope study of recharge processes in the English chalk. J. Hydrol., 101, 31-46.

DAVISSON M.L., PRESSER T.S., CRISS R.E., 1994. Geochemistry of tectonically expelled fluids from the northern Coast ranges, Rumsey Hills, USA. Geochim. Cosmochim. Acta, 58, 1687-1699.

DRAY M., JUSSERAND C., NOVEL J.P., ZUPPI G.M., 1998. Air masse circulation and the isotopic "shadow effect" in precipitation in the French and Italian Alps. IAEA, Vienna, Austria, STI/PUB/1024, 107-117.

FLEISCHER E., GOLDBERG M., GAT J.R., MAGARITZ M., 1977. Isotopic composition of formation waters from deep drillings in Southern Israel. Geochim. Cosmochim. Acta, 41, 511-525.

FONTES J.CH., EDMUNDS W.M., 1989. The use of environmental isotope techniques in arid zone hydrology. A critical review. IHP-III. UNESCO, Paris, France, Technical documents in Hydrology, Project 5.2, $75 \mathrm{pp}$.

GONFIANTINI R., 1978. Standards for stable isotope measurements in natural compounds. Nature, 271, 534-536.

GROSSMANN E.L., UDLUFT W.B., 1991. The extraction of soil water by suctioncup method. A review. J. Soil Sci., 42, 83-93.

HANSEN J., HARRIS H., 1975. Validity of soil water samples collected with ceramic cups. Soil Sci. Soc. Am. Proc., 39, 528-536.

HUTSON J.L., WAGENET R.J., 1992. LEACHM: Leaching Estimation And Chemical Model. Department of Soil, Crop and Atmospheric Sciences. Technical Note $n^{\circ} 92$.

IAEA, 1983. Guidebook on Nuclear Techniques in Hydrology. IAEA, Vienna, Austria, STI/DOC/10/91/2, $439 \mathrm{p}$.

JUSSERAND C., 1980. Extraction de l'eau interstitielle des sédiments et des sols. Comparaison des valeurs de l'oxygène18 par différentes méthodes. Premiers résultats. Catena, 7, 87-96.

LOAGUE K., GREEN R.E., 1991. Statistical and geographical methods for evaluating solute transport models: overview and application. J. Contam. Hydrol., 7, 51-73. 
MASCIOCCO L., PIRASTRU E., SERAZZI M., ZUPPI G.M., 1989. Movimento dell'acqua e trasporto di soluti nella Zona Satura. Compte rendus Symposium "Suolosottosuolo". Torino, Italia, 27-30 septembre 1989, 1, 525-532.

MERLO S, JUSSERAND CI, OLIVE Ph, ZUPPI G.M., 1997. $\mathbf{P}_{\mathrm{CO} 2}$ et minéralisation du carbone dans la Zone Non Saturée du site des eaux minérales de Thonon-les-Bains (Haute-Savoie, France). Bull. Groupe français d'Humidimétrie neutronique et des Techniques associées, 39, 115-120.

NOVEL J.P., RAVELLO M., DRAY M., POLLICINI F., ZUPPI G.M., 1996. Contribution isotopique $\left({ }^{18} \mathrm{O},{ }^{2} \mathrm{H},{ }^{3} \mathrm{H}\right)$ à la compréhension des mécanismes d'écoulement des eaux de surface et des eaux souterraines en vallée d'Aoste (Italie). Geog. Fis. Din. Quat., 18, 315-319.

ROZANSKI K., JOHSEN S.J., SCHOTTERER U., THOMPSON L.G., 1997. Reconstruction of past climates from stable isotope records of paleo-precipitation preserved in continental archives. Hydrol. Sci., 42 (5), 725-745.

SAXENA R.K., DRESSIE Z., 1984. Estimation of grounwater recharge and moisture movement in sandy formations by tracing natural oxygen-18 and injected tritium profiles in the unsaturated zone. Isotope Techniques in Water Resources
Development. IAEA, Vienna, Austria, STI/PUB/650, 139-150.

SHARMA M.L., 1988. Recharge estimation from the depth-distribution of environmental chloride in the unsaturated zone: Western Australia examples. In: Estimation of natural groundwater recharge, SIMMERS I. (Ed.), pp. 159-173.

THORNTHWAITE C.W., MATHER J.R., 1957. Instructions and tables for computing potential evapotranspiration and the water balance. Pubbl. Clim., Drexel Institute, Technical note $n^{\circ} 10,125 \mathrm{p}$.

TRUESDELL A.H., JONES B.F., 1974. WATEQ-a computer program for calculating chemical equilibria of natural waters. J. Res. US Geol. Surv., 2, 233-248.

TURC L., 1961. Évaluation des besoins en eau d'irrigation, évapotranspiration potentielle. Ann. Agr., 23 (1), 13-49.

WALKER G.R., WOODS P.H., ALLISON G.B., 1991. Interlaboratory comparison of methods to determine the stable isotope composition in soil water. Use of Isotope Techniques in Water Resources Development, IAEA, Vienna, Austria, STI/PUB/875, 458-479.

WOODS W.W., PETRAITIS M.J., 1984. Origin and distribution of carbon dioxide in the unsaturated zone of the southern High Plains of Texas. Water Resour. Res., 20,1193-1208. 